\title{
A qualitative systematic review protocol to examine the experiences and needs of informal caregivers of patients with glioma
}

\author{
Ping Yuan ${ }^{1 \#}$, Qiuning Xu ${ }^{1 \#}$, Jinfeng Zhu ${ }^{1,2} \wedge$, Fang Wang ${ }^{1}$, Lixinbei Sheng ${ }^{1}$, Cuiling Ji ${ }^{1}$, Lu Chen ${ }^{1}$ \\ ${ }^{1}$ Department of Neurosurgery, Nanjing Drum Tower Hospital, The Affiliated Hospital of Nanjing University Medical School, Nanjing, China; \\ ${ }^{2}$ Medical School of Nanjing University, Nanjing, China \\ \#These authors contributed equally to this work. \\ Correspondence to: Lu Chen. Department of Neurosurgery, Nanjing Drum Tower Hospital, The Affiliated Hospital of Nanjing University Medical \\ School, Nanjing, China. Email: gycc2011@126.com.
}

\begin{abstract}
Background: Gliomas are the most common primary malignant brain tumors in adults. It has a devastating impact on the cognitive, physical, social, and psychological well-being of patients. Informal caregivers refer to family members, friends, and other carers of the patient who provide unpaid care for patients. They provide physical and psychological support for patients and the family during the disease process. Despite this, there is a paucity of knowledge regarding the experiences and needs of glioma caregivers across the disease trajectory.
\end{abstract}

Methods: A systematic review will be conducted to identify the experiences and needs of informal caregivers of patients with glioma. Seven English databases and four Chinese databases will be analyzed. The search is limited to peer-reviewed full-text articles published either in English or Chinese, with no restrictions on the publication period. According to the Joanna Briggs Institute (JBI) manual for evidence synthesis, two independent reviewers will apply the JBI Critical Appraisal Checklist for Qualitative Research to evaluate the methodological quality of each study. The JBI meta-aggregation method will subsequently be used to synthesize the data, eventually forming themes, categories, and findings.

Discussion: The systematic review is expected to be the first qualitative synthesis of evidence pertains to the experience of family caregivers for glioma patients. The findings generated from the systematic review may be rewarding for researchers to improve care and quality of life for glioma patients and their family members.

Trial registration: PROSPERO CRD42020222307.

Keywords: Glioma; informal caregivers; qualitative research; systematic review

Submitted May 13, 2021. Accepted for publication Jul 14, 2021.

doi: 10.21037/apm-21-1556

View this article at: https://dx.doi.org/10.21037/apm-21-1556

\section{Introduction}

More than 250,000 new cases of primary malignant brain tumors are diagnosed annually worldwide, $77 \%$ of which are gliomas (1). Based on histology and isocitrate dehydrogenase status, gliomas can be divided into four grades according to the World Health Organization, where grades I and II are considered as low-grade, and grades III and IV are considered as high-grade or malignant gliomas (2). Malignant gliomas include anaplastic gliomas (anaplastic oligodendroglioma, anaplastic astrocytoma, and anaplastic oligoastrocytoma) and glioblastoma. As one of

^ ORCID: Qiuning Xu, 0000-0001-8764-2089; Jinfeng Zhu, 0000-0002-0824-4195. 
the most common forms of primary brain tumors in adults, the estimated annual incidence of glioma ranges from 6.6 to 7.1 per 100,000 individuals (3). Gliomas may develop at any age, but its peak incidence is in the fifth and sixth decades of life (4). Although relatively rare, gliomas are life-threatening tumors with a poor prognosis and the 5 -year survival rate is approximately $5 \%$ (5). The median survival of patients with newly diagnosed glioblastoma ranges from less than 1 year to 3 years, with an average of 12-14 months (6). Treatment of malignant glioma consists mainly of surgical resection followed by adjuvant radiation and chemotherapy $(7,8)$.

Despite advances in the medical field, both the treatment and the disease itself can cause damage to patients. Patients with gliomas live with concomitant debilitating symptoms, including functional and cognitive decline, seizures, falls, and personality changes, which have been well documented in the literature (9-12). Therefore, the physical, social, and emotional needs of glioma patients exert an immense burden on their caregivers (13), which is not regularly seen with other cancers $(14,15)$. The terms carer, informal caregiver, and caregiver are used interchangeably in the literature and specifically refer to people who provide unpaid care to a person at home. As there are many concomitant symptoms experienced by patients, the illness perception change plays an important role in determining the psychological distress of informal caregivers. Caregivers' psychological distress is reflected in burden and depressive symptoms during the caring process, which are related to the reduced independence and neuropsychiatric symptoms of the patient (16), moreover, as the disease process, left less time for caregivers to adapt. To be specific, once patients were diagnosed with glioma, the experiences of caregivers were described as being overwhelmed, denial, anger and isolation (17). Then, when it comes to coping with the symptoms of patients, caregivers often described as challenging and struggling to deal with, and may cause anxiety because inadequately prepared for the circumstances (17). After that, they began to take the responsibility as a caregiver for patients, their distress comes from increased responsibility by providing physical care as well as dealing with the neurological symptoms, some caregivers reported feel stressful and relentless (18). At last, when it comes to the end of life care of patients, the caregivers stated they experience death anxiety (19).

Accordingly, the needs of caregivers change vertically in accordance with the patient's condition, caregivers need treatment-related support once patients are diagnosed with glioma, then once patients are discharged home, the psychosocial and supportive care needs go priority. In terms of the end-of life period of patients, emotional needs were identified. It is reported that late-stage caregiving is a difficult and challenging experience at the end of an already burdening treatment trajectory (20). Caregivers were challenged by various physical and psychosocial issues like fatigue, emotional distress (sadness/depressive thoughts) and eventually a reduced work capacity and social network (21). Therefore, there is a need for caregivers to actively share their responsibility and practical tasks with professionals, family, and friends. However, as a systematic review for bereaved glioma caregivers identified that, bereaved caregivers expressed had unmet needs related to the trajectory and their caring role (21). Consequently, they may experience a long and difficult grieving process after patients' death, which implies their needs for psychological support after bereavement. Therefore, more evidence-based practice guidelines and recommendations are warranted is this aspect.

In terms of caregivers coping strategy, a systematic review for caregivers of patients with chronic, advanced illness and end life care showed that a resilient coping style can diminish the risk of stress and burden, and promote adaptation in the caregiver (22). Moreover, as reported in the research, active coping strategies of informal caregivers like problem-solving and social support are positively related to the quality of life (23). Obviously, caregivers play an important role in the outpatient care settings of patients with chronic illnesses and cancers (24), it is essential to develop supportive interventions to prompt coping. In light of this, there has been increasing interest in the experience of caregivers of glioma patients, including qualitative, quantitative, or mixed methodology studies. Given the nature of qualitative research, the unique experience associated with the care of a glioma patient can be understood better by applying qualitative designs. Nevertheless, current studies have examined patient experiences from different perspectives, and it is not possible to establish a comprehensive understanding of the unique experiences from a single study.

Therefore, to gain a deeper understanding of the experience of caregivers looking after glioma patients, this study will systematically review, appraise, and synthesize the current evidence in the literature and provide a set of practical implications for healthcare professionals.

We present the following article in accordance with the PISMA-P reporting checklist (available at https://dx.doi. org/10.21037/apm-21-1556). 


\section{Methods}

This systematic review has been designed as a qualitative meta-synthesis based on guidelines from the Joanna Briggs Institute (JBI) (25) and reported according to the Preferred Reporting Items for Systematic reviews and Meta-Analysis protocol (PRISMA-P) guidelines $(26,27)$. The review is registered with the PROSPERO International Prospective Register of Systematic Reviews (registration number CRD42020222307).

This study focuses on the following two questions:

(I) What are the experiences and views of caregivers for patients with glioma?

(II) What are the support and information needs of caregivers for patients with glioma?

\section{Selection criteria}

\section{Population}

The study cohort includes caregivers of patients with glioma during hospitalization or after discharge, including spouses, parents, adult children, friends, relatives, etc. Participants in the included studies should fulfil the following criteria: participants are 18 years or older; could understand and communicate effectively, and are able to give informed consent. If a qualitative study examined both patients and caregivers, then it can be included only if patients and caregivers are described separately.

\section{Interest of phenomena}

The role of the caregiver brings about life changes, challenges, experiences, needs, coping strategies, etc.

\section{Context}

The entire process of caregiving for patients with glioma during hospitalization or after discharge takes place in either the inpatient or outpatient department.

\section{Design}

A qualitative research design is applied, including but not limited to phenomenology, grounded theory, ethnography, and narrative approaches. Mixed research that contains qualitative methods will also be included.

\section{Exclusion criteria}

The following exclusion criteria will be applied: (I) duplicate studies, abstracts, unavailability of full text article;
(II) reviews and clinical information articles; (III) studies published in languages other than Chinese or English; and (IV) research on child cancer survivors.

\section{Identification of eligible literature}

Following the database search, all identified studies will be collected, and duplicates will be removed. Subsequently, according to the inclusion and exclusion criteria, two independent reviewers will screen the titles and abstracts to assess eligibility. The selection process is summarized in a PRISMA flow diagram (28) (Figure 1).

\section{Search strategy}

A preliminary search of the various databases will be conducted to analyze the keywords and to determine the index terms. The following databases will be searched: MEDLINE, EMBASE, Web of Science, PsycINFO, CINAHL, Cochrane Library, Scopus, China National Knowledge Infrastructure, China Science and Technology Journal Database, Wanfang Database, and Chinese Biomedicine Literature Database. Due to the scarcity of literature, there is no restriction on the publication period. Subsequently, a tailored search strategy will be used on various databases to ensure that all available studies are obtained. Next, the search will be modified according to different databases and will be limited by the language of publication. Reference lists and citations will be manually searched to identify further studies. Searches will be limited to peer-reviewed full-text articles published either in English or Chinese. The following key search terms will be applied: glioma, astrocytoma, caregiver, family, relatives, and qualitative research. All search strategies used in the English databases will be documented with research query and records number. The flow diagram of study identification and selection will be listed in the PRISMA flow diagram (Figure 1). The documents screened and selected in each step will be managed and recorded through the NoteExpress V.3.3.0 software.

\section{Data extraction}

The JBI qualitative data extraction tool (25) will be employed in this process. The extracted data will include specific details about the author, geographic location, setting, participants, objectives, patient's glioma types, interview time and place, phase in the disease trajectory, 


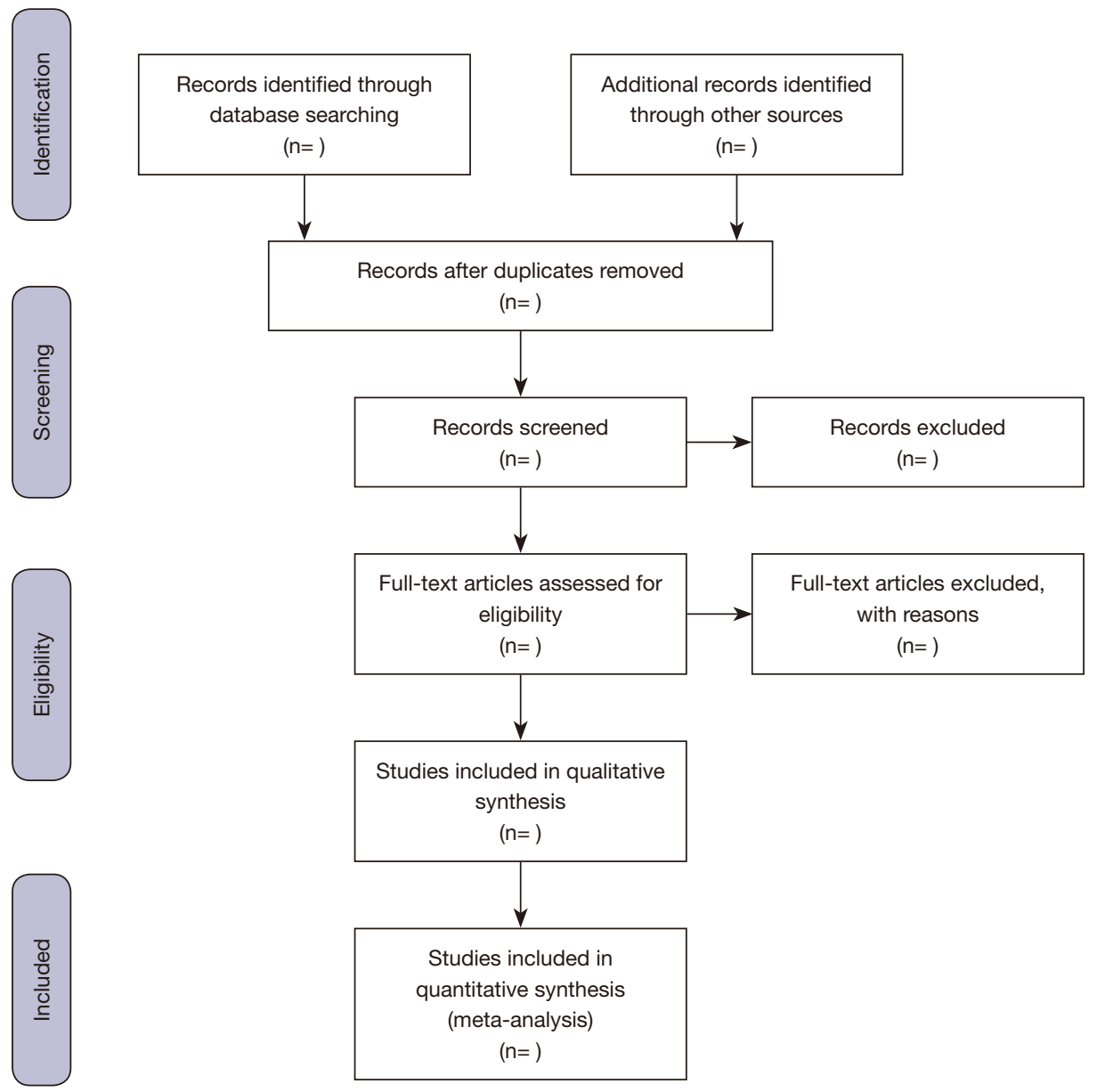

Figure 1 PRISMA flow diagram. From: Moher D, Liberati A, Tetzlaff J, Altman DG, The PRISMA Group. Preferred Reporting Items for Systematic Reviews and Meta-Analyses: The PRISMA Statement. PLoS Med 2009;6:e1000097.

and key findings. If the included study is a mixedmethod study design, only qualitative findings will be analyzed.

\section{Assessment of risk of bias (8)}

The JBI Critical Appraisal Checklist for Qualitative Research (25) will be used to assess the methodological quality of each study. Two researchers will appraise the articles independently, and any disagreements will be resolved by discussion until a consensus is reached.

\section{Statistical analysis}

The JBI meta-aggregative approach will be used for the synthesis of evidence (29). Meta-aggregation is sensitive to the nature and tradition of qualitative research and is predicative on the process of systematic review (30). The approach is also sensitive to the practicality and usability of the primary author's findings and does not seek to re-interpret those findings. Meta-aggregation involves a three-step process:

(I) Extracting findings from all included papers with an accompanying illustration and establishing a level of credibility for each finding;

(II) Developing categories for findings that are sufficiently similar, with at least two findings per category;

(III) Developing one or more synthesized findings of at least two categories.

Two researchers will carry out the process independently to achieve consistency of each synthesized finding. 


\section{Assessing the confidence in the findings}

The synthesized findings will be graded according to the JBI ConQual approach (25) as high, moderate, low, or very low. Each synthesized finding will be appraised along with the type of research, a score for dependability and credibility, and an overall ConQual score (31).

\section{Strengths and limitations of this study}

This systematic review is supposed to be the first research to provide a comprehensive understanding of the experiences of caregivers of patients with glioma. As qualitative research is constantly restrained by the small sample size compared with quantitative research, it is supposed that the synthesizing findings of this systematic review will promote the generalizability of the qualitative findings. Moreover, instruments like JBI Critical Appraisal Checklist for Qualitative Research, JBI ConQual approach and the JBI meta-aggregation method will be applied to establish reliability and validity of this qualitative systematic review. The synthesized findings will be graded according to the JBI ConQual approach as high, moderate, low, or very low. Each synthesized finding will be appraised along with the type of research, a score for dependability and credibility, and an overall ConQual score. We excluded articles published neither in English nor Chinese, and the review findings may not apply to other socio-cultural settings.

\section{Discussion}

In clinical practice, qualitative research contributes significantly to nursing science, particular regarding psychosocial aspects of patient-care, health services provision, policy setting, and health administrations (32). In regard of the glioma patients and their caregivers, they experience a lot of changes in dealing with the disease, which is widely reported in the literature by qualitative research. While caring for someone with a glioma is very demanding due to the dynamic situation of the patients, therefore, it is necessary to investigate caregivers' experience during the whole disease process. This article seeks to expand healthcare processionals' understanding of the complexity of the condition for the glioma patients and their caregivers. It is anticipated that the findings generated by this systematic review will enable health care providers to provide detailed and evidence-based guidance and quality care to patients and their caregivers correspondingly. Lastly, as this systematic review only uses secondary data, ethical approval is not required. No other ethical issues are foreseen. The findings of this systematic review will be published in a peer-reviewed journal and, if possible, presented at scientific conferences. The final published article will report any changes or revisions that arise from the protocol.

\section{Acknowledgments}

Funding: This work was supported by the 15 th batch of "Six Talent Peaks" High-level Talent Selection and Training Funding Program of Jiangsu Province (Grant number: WSW-127) and the Nanjing University of Chinese Medicine "The Construction of Preponderant Disciplines Programme for Institutions of Higher Education in Jiangsu Province" (Grant number: 2019YSHL146).

\section{Footnote}

Reporting Checklist: The authors have completed the PRISMA-P reporting checklist. Available at https://dx.doi. org/10.21037/apm-21-1556

Conflicts of Interest: All authors have completed the ICMJE uniform disclosure form (available at https://dx.doi. org/10.21037/apm-21-1556). The authors have no conflicts of interest to declare.

Ethical Statement: The authors are accountable for all aspects of the work in ensuring that questions related to the accuracy or integrity of any part of the work are appropriately investigated and resolved.

Open Access Statement: This is an Open Access article distributed in accordance with the Creative Commons Attribution-NonCommercial-NoDerivs 4.0 International License (CC BY-NC-ND 4.0), which permits the noncommercial replication and distribution of the article with the strict proviso that no changes or edits are made and the original work is properly cited (including links to both the formal publication through the relevant DOI and the license). See: https://creativecommons.org/licenses/by-nc-nd/4.0/.

\section{References}

1. Walsh KM, Ohgaki H, Wrensch MR. Epidemiology. Handb Clin Neurol 2016;134:3-18.

2. Louis DN, Perry A, Reifenberger G, et al. The 2016 
World Health Organization Classification of Tumors of the Central Nervous System: a summary. Acta Neuropathol 2016;131:803-20.

3. Li K, Lu D, Guo Y, et al. Trends and patterns of incidence of diffuse glioma in adults in the United States, 1973-2014. Cancer Med 2018;7:5281-90.

4. Neuroimmunology Group of Neurology Branch of Chinese Medical Association, Neuroimmunology Committee of Chinese Society for Immunology, Immunology Society of Chinese Stroke Association. Chinese Guidelines for the Diagnosis and Management of Tumefactive Demyelinating Lesions of Central Nervous System. Chinese Medical Journal 2017;130:1838-50.

5. McNeill KA. Epidemiology of Brain Tumors. Neurol Clin 2016;34:981-98.

6. Ostrom QT, Cioffi G, Gittleman H, et al. CBTRUS Statistical Report: Primary Brain and Other Central Nervous System Tumors Diagnosed in the United States in 2012-2016. Neuro Oncol 2019;21:v1-100.

7. Chang L, Su J, Jia X, et al. Treating malignant glioma in Chinese patients: update on temozolomide. Onco Targets Ther 2014;7:235-44.

8. Saito J, Masters J, Hirota K, et al. Anesthesia and brain tumor surgery: technical considerations based on current research evidence. Curr Opin Anaesthesiol 2019;32:553-62.

9. Ownsworth T, Hawkes A, Steginga S, et al. A biopsychosocial perspective on adjustment and quality of life following brain tumor: a systematic evaluation of the literature. Disabil Rehabil 2009;31:1038-55.

10. Baumstarck K, Leroy T, Hamidou Z, et al. Coping with a newly diagnosed high-grade glioma: patientcaregiver dyad effects on quality of life. J Neurooncol 2016;129:155-64.

11. GBD 2016 Brain and Other CNS Cancer Collaborators. Global, regional, and national burden of brain and other CNS cancer, 1990-2016: a systematic analysis for the Global Burden of Disease Study 2016. Lancet Neurol 2019;18:376-93.

12. Zwinkels H, Dirven L, Vissers T, et al. Prevalence of changes in personality and behavior in adult glioma patients: a systematic review. Neurooncol Pract 2016;3:222-31.

13. Halkett GK, Lobb EA, Shaw T, et al. Do carer's levels of unmet needs change over time when caring for patients diagnosed with high-grade glioma and how are these needs correlated with distress? Support Care Cancer 2018;26:275-86.
14. Given BA, Given CW, Sherwood P. The challenge of quality cancer care for family caregivers. Semin Oncol Nurs 2012;28:205-12.

15. Drageset S, Lindstrøm TC, Underlid K. Coping with breast cancer: between diagnosis and surgery. J Adv Nurs 2010;66:149-58.

16. Sterckx W, Coolbrandt A, Dierckx de Casterlé B, et al. The impact of a high-grade glioma on everyday life: a systematic review from the patient's and caregiver's perspective. Eur J Oncol Nurs 2013;17:107-17.

17. Schubart JR, Kinzie MB, Farace E. Caring for the brain tumor patient: family caregiver burden and unmet needs. Neuro Oncol 2008;10:61-72.

18. McConigley R, Halkett G, Lobb E, et al. Caring for someone with highgrade glioma: a time of rapid change for caregivers. Palliat Med 2010;24:473-79.

19. Adelbratt S, Strang P. Death anxiety in brain tumour patients and their spouses. Palliat Med 2000;14:499-507.

20. Piil K, Mary J. Bereaved Caregivers to Patients With High-Grade Glioma: A Qualitative Explorative Study. J Neurosci Nurs 2018;50:94-9.

21. Piil K, Nordentoft S, Larsen A, at al. Bereaved caregivers of patients with high-grade glioma: a systematic review. BMJ Support Palliat Care 2019;9:26-33.

22. Palacio GC, Krikorian A, Gómez-Romero MJ, et al. Resilience in Caregivers: A Systematic Review. Am J Hosp Palliat Care 2020;37:648-58.

23. Baumstarck K, Chinot O, Tabouret E, et al. Coping strategies and quality of life: a longitudinal study of highgrade glioma patient-caregiver dyads. Health Qual Life Outcomes 2018;16:157.

24. Adashek JJ, Subbiah IM. Caring for the caregiver: a systematic review characterising the experience of caregivers of older adults with advanced cancers. ESMO Open 2020;5:e000862.

25. Aromataris E, Munn Z (Editors). JBI Manual for Evidence Synthesis. JBI 2020. Available online: https://doi. org/10.46658/JBIMES-20-01.

26. Moher D, Shamseer L, Clarke M, et al. Preferred reporting items for systematic review and meta-analysis protocols (PRISMA-P) 2015 statement. Syst Rev 2015;4:1.

27. Shamseer L, Moher D, Clarke M, et al. Preferred reporting items for systematic review and meta-analysis protocols (PRISMA-P) 2015: elaboration and explanation. BMJ 2015;350:g7647.

28. Moher D, Liberati A, Tetzlaff J, et al. Preferred reporting items for systematic reviews and meta-analyses: the PRISMA statement. PLoS Med 2009;6:e1000097. 
29. Pearson A. Balancing the evidence: incorporating the synthesis of qualitative data into systematic reviews. JBI Reports 2004;2:45-64.

30. Lockwood C, Munn Z, Porritt K. Qualitative research synthesis: methodological guidance for systematic reviewers utilizing meta-aggregation. Int J Evid Based Healthc 2015;13:179-87.

31. Munn Z, Porritt K, Lockwood C, et al. Establishing

Cite this article as: Yuan P, Xu Q, Zhu J, Wang F, Sheng L, Ji C, Chen L. A qualitative systematic review protocol to examine the experiences and needs of informal caregivers of patients with glioma. Ann Palliat Med 2021;10(7):8310-8316. doi: 10.21037/ apm-21-1556 confidence in the output of qualitative research synthesis: the ConQual approach. Bmc Med Res Methodol 2014;14:108.

32. Leung L. Validity, reliability, and generalizability in qualitative research. J Family Med Prim Care 2015;4:324-7.

(English Language Editor: J. Teoh) 